\title{
Changes in 2'-deoxycytidine levels in various tissues of tumor-bearing mice
}

\author{
AYANO IWAZAKI, KIMIE IMAI, KUNIO NAKANISHI and MASANORI YOSHIOKA \\ Faculty of Pharmaceutical Sciences, Setsunan University, Osaka 573-0101, Japan
}

Received July 12, 2010; Accepted August 25, 2010

DOI: $10.3892 / \mathrm{ol} .2010 .170$

\begin{abstract}
The nucleoside 2'-deoxycytidine (dCyd) increases in the plasma of cancer patients with poor prognoses. 5-fluorouracil (5FU) is one of the anti-cancer agents used in chemotherapy for patients whose plasma dCyd is elevated. We examined the free dCyd level in various tissues of mice, with and without tumors, and in mice with and without the administration of $5 \mathrm{FU}$ or of $\mathrm{dCyd}$, and investigated the effects of $\mathrm{dCyd}$ in tumor-bearing animals. SP2/0-Ag14 mouse myeloma cells were transplanted subcutaneously into mice and 5FU or dCyd was administered intraperitoneally. Free dCyd was measured in blood and tissues by HPLC at two time points, once when mouse body weight was maximally decreased (1 day after the last administration of 5FU, day 16) and again when it returned to control level at 1 week after the last 5FU treatment (day 22). Results showed that in tumor-bearing mice, the level of dCyd (per $g$ wet weight) increased in the spleen. The change in liver weight caused by the administration of $5 F U$ correlated with the level of dCyd in the liver. Notably, the relative tumor volume and tumor weight was decreased in dCyd-treated animals in comparison with controls. In conclusion, the levels of dCyd were markedly altered in the tissues of the reticuloendothelial and lymphatic systems, such as liver and spleen, and dCyd apparently had the ability to inhibit tumor growth in the body.
\end{abstract}

\section{Introduction}

2'-Deoxycytidine (dCyd), a pyrimidine nucleoside, is found at high concentrations in the plasma of poor-prognosis patients with bladder cancer, acute lymphoma (1) or hepatoma (2), and of breast cancer patients who have been treated with chemotherapy [cyclophosphamide, methotrexate and 5-fluorouracil (5FU)] (3). Moreover, the anti-tumor activity of 5FU has been found to be decreased by dCyd in SP2/0-Ag14 mouse myeloma-bearing mice (4). However, it is unclear why dCyd

Correspondence to: Dr Ayano Iwazaki, Faculty of Pharmaceutical Sciences, Setsunan University, 45-1 Nagaotoge-cho, Hirakata, Osaka 573-0101, Japan

E-mail: ayano@pharm.setsunan.ac.jp

Key words: 5-fluorouracil, spleen, tumor volume increases in cancer patients and whether the administration of $5 F U$ affects the dCyd level in tissues. Thus, we examined free $\mathrm{dCyd}$ levels in non-tumor-bearing and tumor-bearing mouse tissues, and in tumor-bearing mice treated with 5FU or dCyd.

Moyer et al (5) studied the metabolism of radiolabeled pyrimidine nucleosides administered to mice by intravenous injection. They found that the half-life of dCyd was less than $9 \mathrm{~min}$ and that deoxyuridine (dUrd), from deamination of $\mathrm{dCyd}$, was present even at $1 \mathrm{~min}$, and equaled the concentration of dCyd in the plasma by $10 \mathrm{~min}$. Additionally, they measured the radioactivity in the acid-soluble fractions of tissues, as a nucleoside or in the DNA fraction, after $30 \mathrm{~min}$. The tissue concentration of unmetabolized dCyd was very low. This study suggested that administered dCyd was removed from the plasma almost immediately and was distributed in tissues in the form of dUrd and/or DNA.

This report examined endogenous-free dCyd levels in various tissues of mice into which SP2/0 cells had been subcutaneously transplanted to generate solid-tumors, with or without 5FU or dCyd administration. The free dCyd level was found to be particularly elevated in the spleens of tumorbearing animals. Administration of 5FU was observed to affect liver weight and this alteration may involve changes in the free dCyd level.

\section{Materials and methods}

Chemicals. dCyd and 5FU were obtained from Sigma-Aldrich (St. Louis, MO, USA). RPMI-1640 medium (RPMI) was obtained from Nikken Biomedical Laboratory (Kyoto, Japan). Fetal bovine serum (FBS) was from Sanko Junyaku (Tokyo, Japan). Plastic tissue culture dishes, 100-mm in diameter, were from IWAKI Co. (Tokyo, Japan). Penicillin and streptomycin were obtained from Meiji Seika Co. (Tokyo, Japan). Phosphatebuffered saline (PBS) was from Takara Shuzo Co., Ltd. (Shiga, Japan). Trypan blue and trichloroacetic acid (TCA) were from Wako Pure Chemical Ind., Ltd. (Osaka, Japan). Filter units (Millex $^{\mathrm{TM}}-\mathrm{GV} 0.22-\mu \mathrm{m}$, sterile and Millex ${ }^{\mathrm{TM}}-\mathrm{HV} 0.45-\mu \mathrm{m}$, nonsterile) were purchased from Millipore Corporation (Bedford, MA, USA). Glycine, acetonitrile, and EDTA.2Na were obtained from Nacalai Tesque (Kyoto, Japan) and trifluoroacetic acid (TFA) was from Merck KGaA (Darmstadt, Germany).

Cell cultures of SP2/0 cells. SP2/0 cells were obtained from the Collection of Cancer Cell Lines (National Institute of 


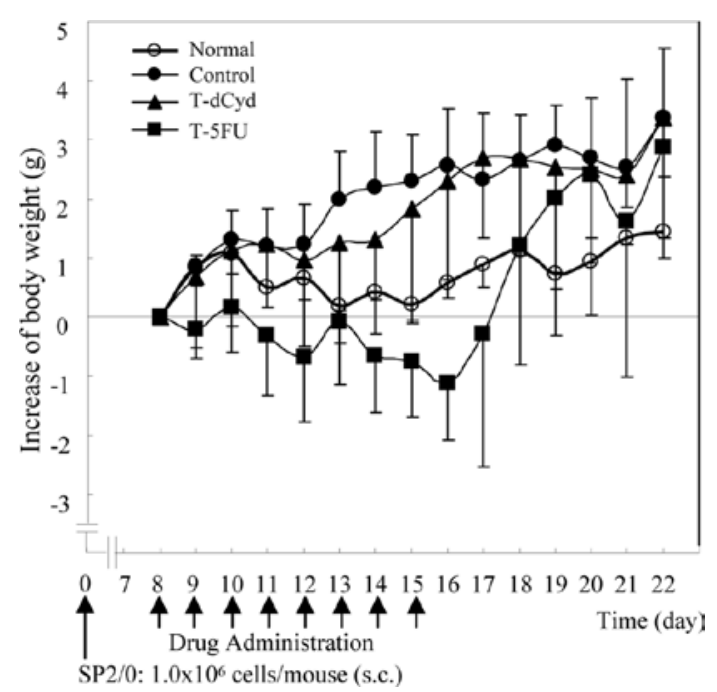

Figure 1. Effect of 5FU and dCyd on the body weight of mice.

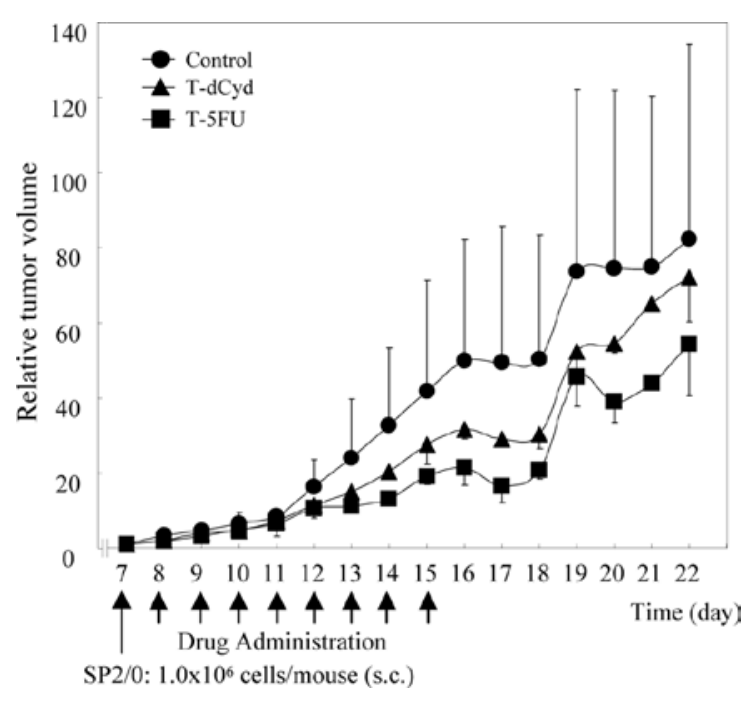

Figure 2. Effect of 5FU and dCyd on the relative tumor volume.
Hygienic Science, Tokyo, Japan). These cells were maintained in culture dishes in RPMI-1640 containing 10\% heat-inactivated FBS, penicillin at $100 \mathrm{U} / \mathrm{ml}$ and streptomycin at $100 \mu \mathrm{U} / \mathrm{ml}$, under a humidified atmosphere of $5 \% \mathrm{CO}_{2}$ at $37^{\circ} \mathrm{C}$. To inoculate mice, the cells were removed from dishes by pipetting and washed twice with PBS. The cell number was determined during trypan blue exclusion tests (6).

Animals and dCyd and 5FU treatments. BALB/c mice (male, 5-week-old, weighing 20-25 g) were obtained from Japan SLC (Hamamatsu, Japan). The animals were continually cared for in a room maintained at $25 \pm 1^{\circ} \mathrm{C}$ and $55 \pm 5 \%$ humidity with free access to water and food. For tumor-bearing treatment groups, SP2/0 cell suspension ( $1 \times 10^{6}$ cells/mouse) was inoculated subcutaneously (s.c.) into a shaved area on the back of each mouse on day 0 . Then, 7 days later, body weight and relative tumor volume were measured. Tumor volume was calculated using the following formula: tumor volume $\left(\mathrm{mm}^{3}\right)=$ $\mathrm{A} \times \mathrm{A} \times \mathrm{B} / 2$, where $\mathrm{A}$ is the smallest diameter $(\mathrm{mm})$ and $\mathrm{B}$ is the largest diameter $(\mathrm{mm})$ of solid tumor, measuring with a vernier caliper. When tumor volume in these animals reached approximately $100-300 \mathrm{~mm}^{3}$ at day 7 , all mice were divided into five groups as follows: normal (PBS administered to nontumor-bearing mice), non-tumor-dCyd ( $\mathrm{N}-\mathrm{dCyd}, 0.1 \mathrm{mmol} / \mathrm{kg}$ $\mathrm{dCyd}$ administered to non-tumor-bearing mice), control (PBS administered to tumor-bearing mice), tumor-dCyd (T-dCyd, $0.1 \mathrm{mmol} / \mathrm{kg} \mathrm{dCyd}$ administered to tumor-bearing mice) and tumor-5FU (T-5FU, $0.15 \mathrm{mmol} / \mathrm{kg} 5 \mathrm{FU}$ administered to tumor-bearing mice). Moreover, each group was divided into two samples and sacrificed on different days as described below. Each sample consisted of 2-5 mice. 5FU or dCyd was dissolved in sterile PBS at a given concentration and filtered through a $0.22-\mu \mathrm{m}$ filter. On days 8 through 15 , PBS, dCyd or $5 \mathrm{FU}$ was administered at $0.1 \mathrm{ml} / 10 \mathrm{~g}$ body weight by intraperitoneal injection (i.p.), and body weight and relative tumor volume were recorded. Relative tumor volume was calculated by dividing the tumor volume on a given day by that on day 7. Change in body weight was calculated as the weight on a given day minus the weight on the first day of treatment administration (day 8). Animals in one sample of each group were sacrificed at 1 day after the final i.p. injection (day 16), and the remaining animals were sacrificed at 6 days after the final i.p. injection (day 22), under diethyl ether anesthesia. Blood was collected from the heart into test tubes containing EDTA $(1.5 \mathrm{mg} / \mathrm{ml}$ blood) and body tissues were removed and weighed.

The protocol was performed according to the guidelines of the Japanese Society for Pharmacology and was approved by the Committee for Ethical Use of Experimental Animals at Setsunan University.

Measurement of dCyd in tissues by high-performance liquid chromatography (HPLC). TCA (10 $\mathrm{ml}$ of a $10 \%$ solution) was added to $1 \mathrm{~g}$ (wet weight) of tissue, and the tissue was homogenized. Plasma samples were mixed with equal volumes of $10 \%$ TCA. Tissue homogenate and plasma were centrifuged at $2,000 \mathrm{x}$ for $15 \mathrm{~min}$. The supernatant was neutralized with $2 \mathrm{M}$ $\mathrm{KOH}$ and applied to Supelclean LC-SAX SPE tubes (Supelco, Bellefonte, PA, USA) that had been conditioned by washing with distilled water and $0.2 \mathrm{M}$ glycine buffer ( $\mathrm{pH}$ 3.0). dCyd was eluted with the glycine buffer, passed through a $0.45-\mu \mathrm{m}$ filter and subjected to HPLC. An aliquot of the filtrate was applied to a MIGHTYSIL RP-18 GP Aqua 250-4.6 (5 $\mu \mathrm{m})$ column (Kanto Kagaku Co., Tokyo, Japan). The column was maintained at $40^{\circ} \mathrm{C}$. dCyd was eluted with a linear gradient from $0.1 \%$ TFA to $20 \%$ acetonitrile containing $0.1 \%$ TFA, at a rate of $1.0 \mathrm{ml} / \mathrm{min}$ for $30 \mathrm{~min}$. The eluate was monitored with a UV detector (875-UV detector; Jasco, Tokyo, Japan) at $265 \mathrm{~nm}$. The complete instrument consisted of an 880-PU pump (Jasco), 880-51 degasser (Jasco), LG-980-02 ternary gradient unit (Jasco), 860-CO column oven and Unicorder U-228 (Pantos, Uji, Japan).

Statistical analysis of measurements. Data were analyzed using the F-test for variance and Student's t-test for significance.

\section{Results}

Effects of drug administration on body weight and relative tumor volume. Body weight in the tumor-bearing control 
A
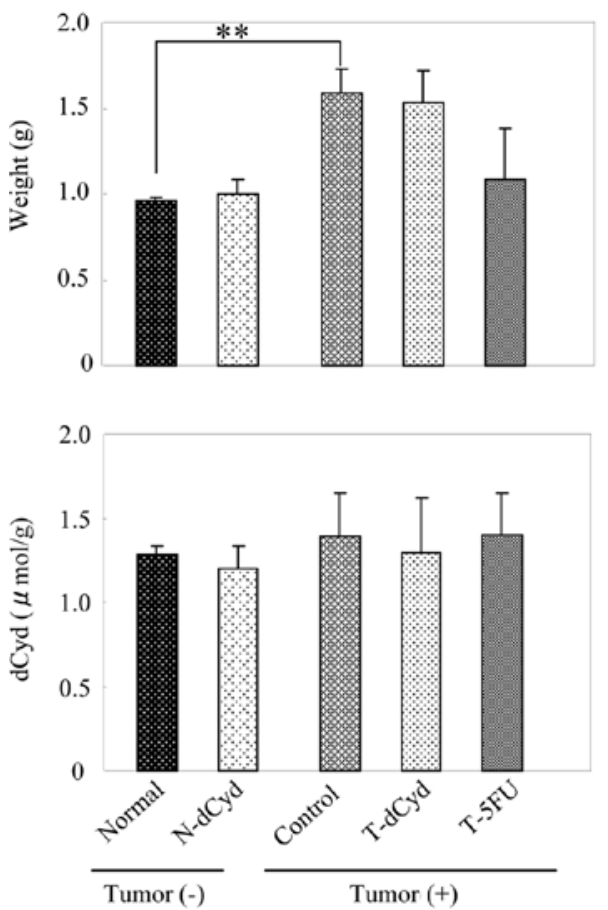

B
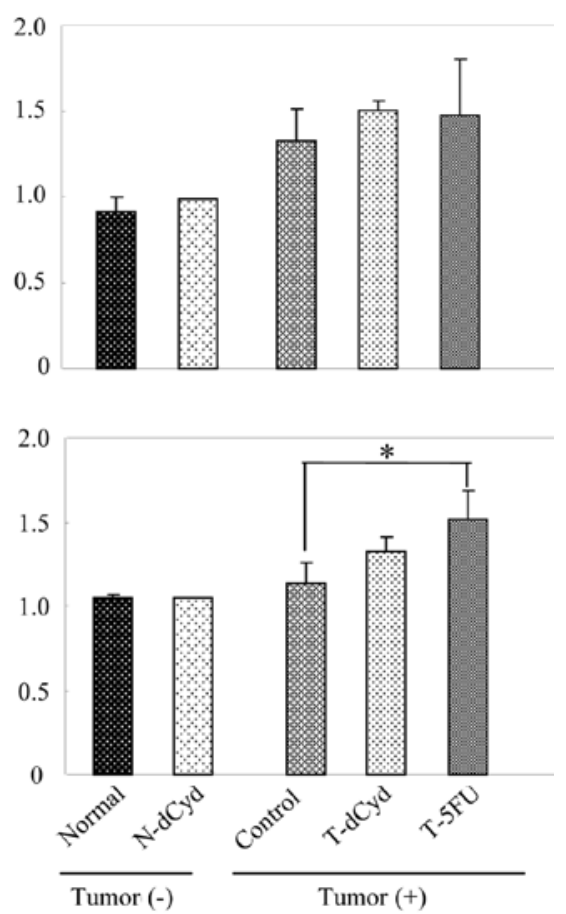

Figure 3. Liver weight and dCyd levels at days 16 (A) and 22 (B). Significant differences from normal (non-tumor-bearing controls) are indicated by * $<0.05$ and ${ }^{* *} \mathrm{P}<0.01$.

A
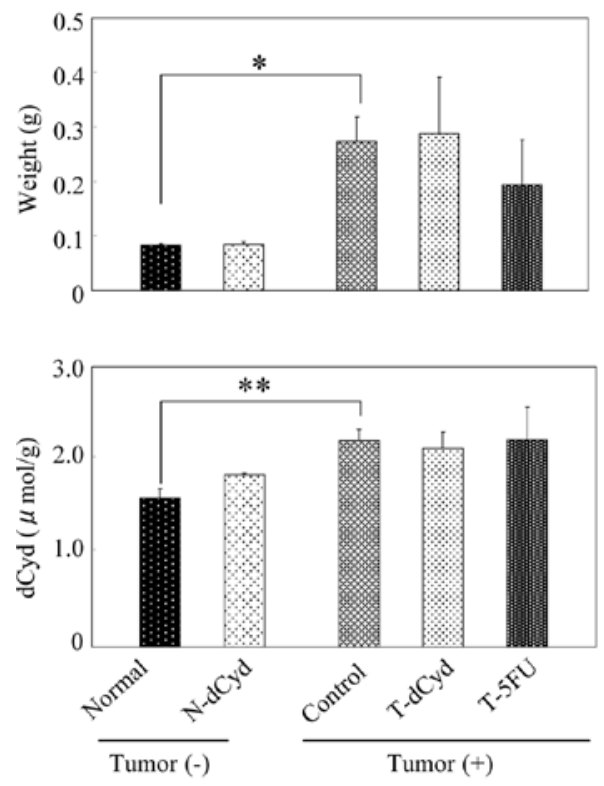

B
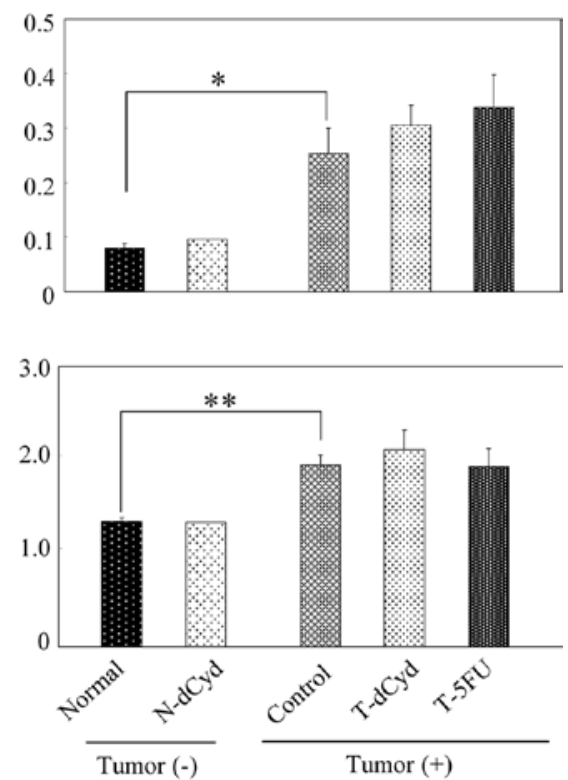

Figure 4. Spleen weight and dCyd levels at days 16 (A) and 22 (B). Significant differences from normal (non-tumor-bearing controls) are indicated by ${ }^{*} \mathrm{P}<0.05$ and ${ }^{* *} \mathrm{P}<0.01$.

group was heavier than in the normal group (Fig. 1). In the T-5FU group, body weight at day 16 was lower than the baseline, but increased to the level of the tumor-bearing controls at day 22. Body weight in the $\mathrm{N}$-dCyd group was similar to that in the non-tumor-bearing normal group (data not shown).

The relative tumor volume of the tumor-bearing control group was 50 on day 16 , while that of the T-dCyd group was 30 (Fig. 2). In the T-5FU group, the relative tumor volume increased less than in the control group.

Tissue weight and dCyd level. At day 16, liver weight in the tumor-bearing control group was 1.4-fold heavier than in the normal group (Fig. 3A). However, liver weight of the T-5FU group decreased in comparison to its control group. The level of dCyd in the liver was not affected by tumor-bearing status 
A
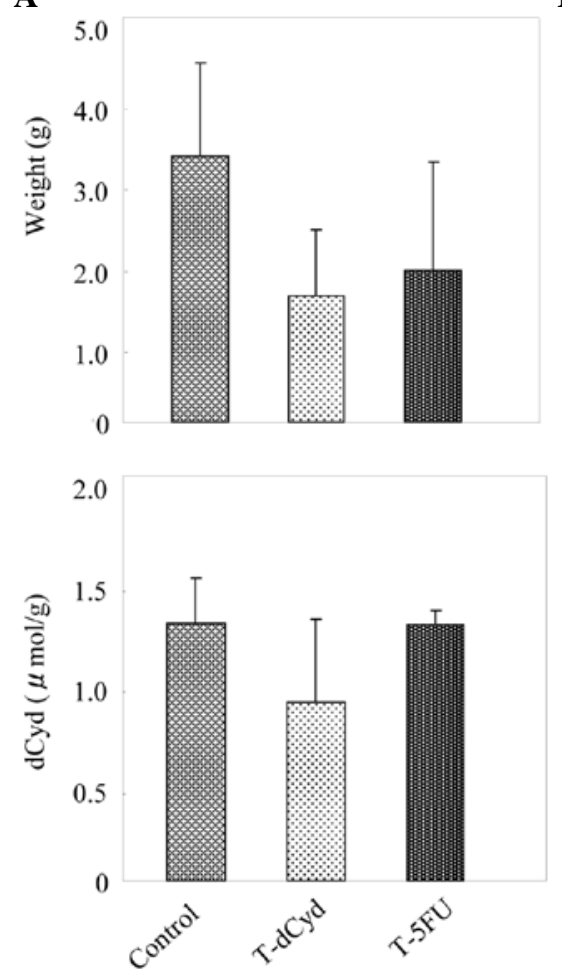

B
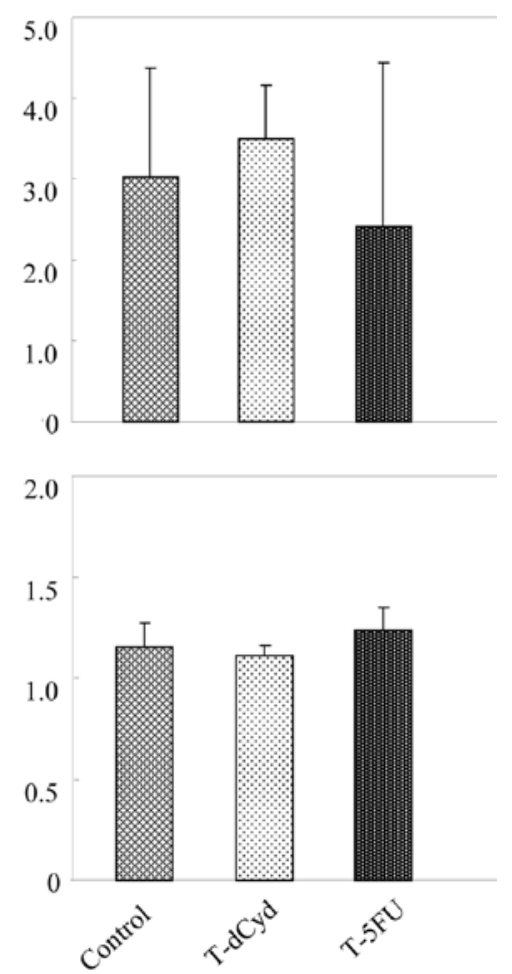

Figure 5. Tumor weight and dCyd levels at days 16 (A) and 22 (B).

A

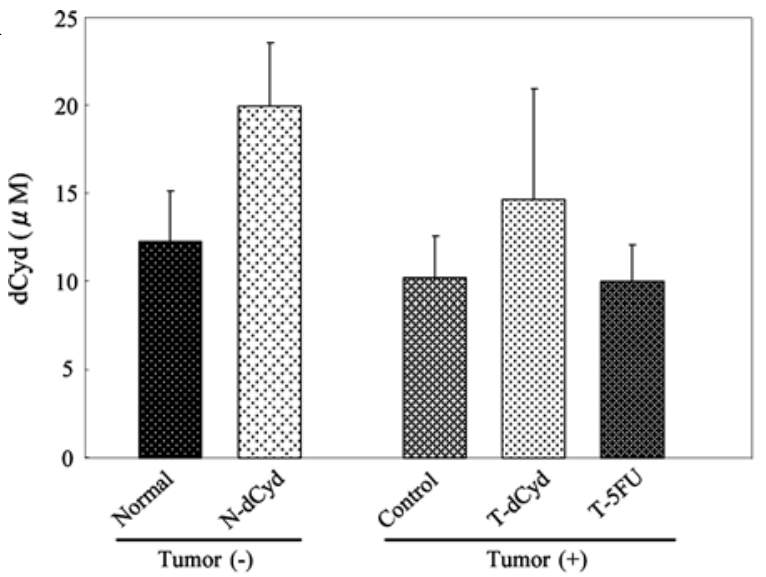

B

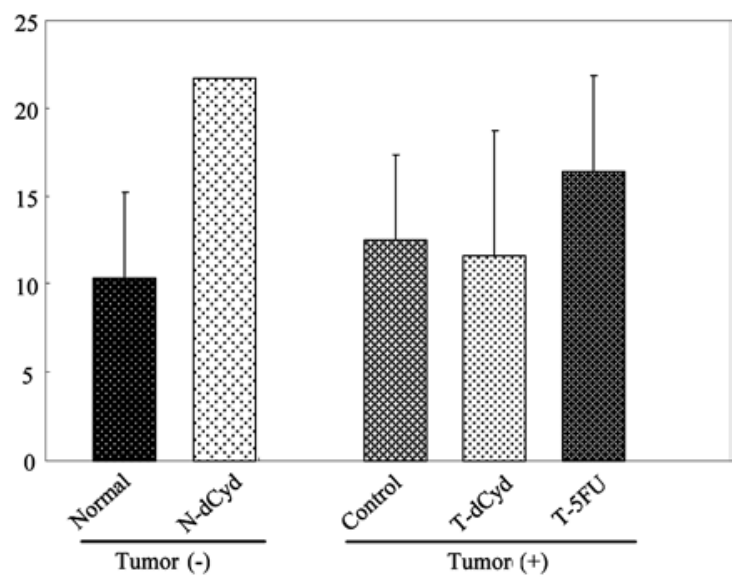

Figure 6. dCyd concentrations in plasma at days 16 (A) and 22 (B).

or by administration of dCyd or $5 \mathrm{FU}$. At day 22 , liver weight in the tumor-bearing control group was 1.6-fold heavier than in the normal group (Fig. 3B), and that in the T-5FU group was similar to the controls. The level of dCyd in the liver was increased by administration of $5 \mathrm{FU}$.

At day 16, spleen weight and the level of dCyd in the spleen of the tumor-bearing control group increased 4.3-fold, 1.4-fold above the normal group (Fig. 4A). At day 22, spleen weight and the level of dCyd in the spleen of the tumor-bearing control group was increased 4.2-fold, 1.4-fold above the normal group (Fig. 4B). Administration of dCyd did not affect the level of dCyd detected in the liver or spleen of tumor-bearing or nontumor-bearing mice on either day.

At day 16, tumor weight appeared to decrease in the T-dCyd and T-5FU groups (Fig. 5A). The level of dCyd was similar among the groups. At day 22, tumor weights and levels of dCyd in tumors were unaffected by the administration of dCyd or 5FU (Fig. 5B). At day 16, the level of dCyd in the plasma was approximately $10 \mu \mathrm{M}$ and was increased by administration of dCyd (Fig. 6A). No difference in plasma dCyd was observed between tumor-bearing and non-tumorbearing mice on days 16 and 22 (Fig. 6A and B).

Table I shows the weight, level of dCyd and net amount of dCyd in various tissues of the normal and control groups on days 16 and 22. The weights of kidney and lungs were not different between groups. On the other hand, the weights of liver and spleen were markedly increased in the tumor-bearing control group. The level of dCyd in liver, kidney, spleen and tumor was approximately $1.0-2.0 \mu \mathrm{mol} / \mathrm{g}$ tissue, whereas that in the heart and lungs was $0.2-0.8 \mu \mathrm{mol} / \mathrm{g}$ tissue. The net 
Table I. dCyd levels in various tissues and plasma of BALB/c mice, at days 16 and 22 after inoculation with (+) or without (-) SP2/0 mouse myeloma cells.

\begin{tabular}{|c|c|c|c|c|c|c|c|}
\hline \multirow{3}{*}{ Tissue } & \multirow{3}{*}{ Tumor } & & & \multicolumn{4}{|c|}{ dCyd } \\
\hline & & \multicolumn{2}{|c|}{ Tissue weight (g) } & \multicolumn{2}{|c|}{ Level $(\mu \mathrm{mol} / \mathrm{g})$} & \multicolumn{2}{|c|}{$\begin{array}{l}\text { Net amount } \\
(\mu \mathrm{mol} / \mathrm{tissue})\end{array}$} \\
\hline & & 16th & $22 \mathrm{nd}$ & 16 th & $22 \mathrm{nd}$ & 16th & 22nd \\
\hline \multicolumn{8}{|l|}{ Heart } \\
\hline & - & 0.109 & 0.111 & 0.617 & 0.418 & 0.067 & 0.046 \\
\hline & + & 0.107 & $0.092^{\mathrm{a}}$ & 0.764 & 0.464 & 0.062 & 0.043 \\
\hline \multicolumn{8}{|l|}{ Liver } \\
\hline & - & 0.966 & 0.912 & 1.269 & 1.045 & 1.245 & 0.952 \\
\hline & + & $1.593^{b}$ & 1.328 & 1.369 & 1.133 & 2.229 & 1.510 \\
\hline \multicolumn{8}{|l|}{ Kidney } \\
\hline & - & 0.152 & 0.149 & 1.815 & 1.213 & 0.275 & 0.179 \\
\hline & + & 0.168 & 0.152 & 1.724 & 1.345 & 0.290 & 0.205 \\
\hline \multicolumn{8}{|l|}{ Lung } \\
\hline & - & 0.142 & 0.136 & 0.188 & 0.410 & 0.027 & 0.056 \\
\hline & + & 0.158 & 0.147 & 0.248 & 0.424 & 0.040 & 0.062 \\
\hline \multicolumn{8}{|l|}{ Spleen } \\
\hline & - & 0.064 & 0.060 & 1.594 & 1.344 & 0.133 & 0.107 \\
\hline & + & $0.273^{\mathrm{a}}$ & $0.254^{\mathrm{a}}$ & $2.212^{\mathrm{b}}$ & $1.947^{\mathrm{b}}$ & $0.605^{\mathrm{b}}$ & $0.492^{\mathrm{b}}$ \\
\hline Tumor & + & 3.279 & 3.020 & 1.271 & 1.154 & 4.050 & 3.546 \\
\hline \multicolumn{8}{|l|}{ Plasma } \\
\hline & - & & & 12.3 & 10.3 & & \\
\hline & + & & & 10.2 & 12.5 & & \\
\hline
\end{tabular}

Significant differences from normal (non-tumor-bearing controls) are indicated by ${ }^{\mathrm{a}} \mathrm{P}<0.05$ and ${ }^{\mathrm{b}} \mathrm{P}<0.01$.

amount of dCyd in liver and spleen was markedly increased in the control group.

\section{Discussion}

We investigated the free dCyd level in various tissues of mice, with and without tumors, and in mice with and without 5FU or dCyd administration. The free dCyd level was measured at 1 day after the last drug administration (day 16), when the body weight of mice administered 5FU was maximally decreased, and at 1 week after the last administration (day 22), when weights had returned to the level of the controls. It is likely that the increasing body weight was influenced by increasing tumor volumes.

Liver weights had decreased with 5FU treatment at day 16 and were similar to those of the tumor-bearing control group at day 22. At day 22, dCyd in liver in the T-5FU group was higher than in the control group; this difference appeared to correlate with the increase in plasma dCyd.

Berlinger (7) reported that the concentration of $\mathrm{dCyd}$ was increased in plasma and in cerebrospinal fluid after hepatectomy. Schneider et al (8) reported that the activity of the enzyme that synthesizes dCyd diphosphate choline was increased in rat hepatomas and in regenerating liver after partial hepatectomy and was related to increased DNA synthesis. These reports may be significant for our study, in which the level of dCyd in the liver increased with the recovery of liver weight after decreases caused by administration of the anti-tumor agent 5FU. Thus, it is suggested that the free dCyd level is influenced by changes in tissue weight or tissue regeneration.

In this study, spleen had the highest level of dCyd among the measured tissues. In spleen, the weight, level of dCyd and net amount of dCyd were all significantly increased in tumor-bearing mice, compared to the non-tumor groups, on both day 16 and day 22. Osogoe et al reported that after tritiated dCyd was administered to mice intraperitoneally, radioactivity was strongly detected in the germinal center cells of the spleen and in Peyer's patches of the intestine in both mouse (9) and rat (10). We speculate whether dCyd may act as a defense mechanism in the body, given the spleen's role among the lymphatic tissues and its involvement in the reticuloendothelial system. The spleen contains plasma cells, B lymphocytes, T lymphocytes, antigen-presenting cells and macrophages, which all function in immunity. Recent studies have focused on activation-induced cytidine deaminase (AID), a member of the cytidine-deaminase family, for which cytidine and dCyd are substrates. AID is essential for somatic hypermutation (SHM) and class-switch recombination in the immunoglobulin genes (11), and the AID gene is specifically 
expressed in germinal center B cells (12) in the spleen, in its role as a secondary lymphoid organ. Moreover, the inappropriate expression of AID causes DNA and/or RNA editing $(13,14)$ and is involved in human malignancies via genomic DNA cleavage, which contributes to tumorigenesis (15). The increase in free dCyd in the body may inhibit mutations caused by AID through an effect on the production or repair of DNA and/or RNA.

Of the tissues measured, the highest net amount of dCyd was found in tumors. We think this high level reflects the dCyd level in plasma, where it can be used as prognostic marker for cancer patients (1). However, the dCyd level in plasma was unaffected by tumor-bearing status in this study. These results suggest that the dCyd level in plasma depends indirectly on tumor size. For example, it is possible that dCyd level is affected by changes in the activity of enzymes that metabolize dCyd. We aim to examine the activities of such enzymes, e.g., deoxycytidine deaminase (16), cytidine deaminase (17) and deoxycytidine kinase $(18,19)$, in tissues of non-tumor-bearing and tumor-bearing mice.

Notably, the administration of dCyd to tumor-bearing mice seemed to decrease the relative tumor volume and the tumor weight in this study. This suggests that dCyd has the ability to inhibit tumor growth, at least somewhat. However, in our previous study (4), dCyd had no effect on survival in tumor-bearing mice. Differences between the previous and the present study in the tumor inoculation protocol may be responsible for this. In the previous experiments, the tumor was implanted and dCyd was administered intraperitoneally, whereas in the present study, the tumor was implanted subcutaneously on the back of the mice, with dCyd administered intraperitoneally. Since dCyd must travel in the blood circulation to reach the solid tumor, metabolites or anabolites of $\mathrm{dCyd}$ may have anti-tumor activity. Additionally, the ability of dCyd to inhibit tumor growth may be indirect (e.g., by activating defensive factors in lymphatic tissues).

From the results of this study, we found that i) the administration of 5FU had an effect on liver weight, which correlated with tissue dCyd level, ii) free dCyd levels were high in the spleens of tumor-bearing mice and iii) the administration of dCyd had the ability to inhibit solid tumor growth. Further studies are required to clarify the role of $\mathrm{dCyd}$ in the tumorbearing body.

\section{Acknowledgements}

The authors thank Ms. R. Ikenaga for the technical assistance.

\section{References}

1. Ahmed WA, Ali-Din NH, Yoshioka M and EL-Merzabani M: 2'-Deoxycytidine (DCYD) as a potential biological marker for detecting acute lymphocytic leukemia and bladder cancer. J Union Arab Biol Cairo 20A: 97-111, 2003.

2. Ahmed WA, Moneer M, Abo-Shady MM, Mansour HH, Abd-El-Wahab N, Yoshioka M and EL-Merzabani M: 2'-Deoxycytidine as a potential biomarker for detection of hepatocellular carcinoma. Egyptian J Hosp Med 21: 191-201, 2005.
3. Yoshioka M, Abu-Zeid M, Kubo $\mathrm{T}$ and El-Merzabani M: Identification of a previously unknown compound as 2'-deoxycytidine found in the plasma of breast cancer patients under combined chemotherapy. Biol Pharm Bull 17: 169-174, 1994.

4. Iwazaki A and Yoshioka M: 2'-Deoxycytidine decreases the anti-tumor effects of 5-fluorouracil on mouse myeloma cells. Biol Pharm Bull 33: 1024-1027, 2010.

5. Moyer JD, Malinowski N and Ayers O: Salvage of circulating pyrimidine nucleosides by tissues of the mouse. J Biol Chem 260: 2812-2818, 1985.

6. Rossi L, Serafini S, Schiavano GF, Casabianca A, Vallanti G, Chiarantini L and Magnani M: Metabolism, mitochondrial uptake and toxicity of 2', 3'-dideoxycytidine. Biochem J 344: 915-920, 1999.

7. Berlinger WG, Stene RA, Spector R and Al-Jurf AS: Plasma and cerebrospinal fluid nucleosides and oxypurines in acute liver failure. J Lab Clin Med 110: 137-144, 1987.

8. Schneider WC and Behki RM: Phosphorus compounds in animal tissues. VII. Enzymatic formation of deoxycytidine diphosphate choline and lecithin by tissue homogenates. J Biol Chem 238: 3565-3571, 1963.

9. Osogoe B and Ueki A: A radioautographic study of the utilization of deoxycytidine for the formation of deoxyribonucleic acid-thymine in lymphocytes. J Cell Biol 46: 403-405, 1970.

10. Osogoe B, Tyler RW and Everett NB: The patterns of labeling of germinal-center cells with tritiated deoxycytidine. J Cell Biol 57: 215-220, 1973.

11. Muramatsu M, Kinoshita K, Fagarasan S, Yamada S, Shinkai Y and Honjo T: Class switch recombination and hypermutation require activation-induced cytidine deaminase (AID), a potential RNA editing enzyme. Cell 102: 553-563, 2000.

12. Muramatsu M, Sankaranand VS, Anant S, Sugai M, Kinoshita K, Davidson NO and Honjo T: Specific expression of activationinduced cytidine deaminase (AID), a novel member of the RNA-editing deaminase family in germinal center B cells. J Biol Cell 274: 18470-18476, 1999.

13. Kotani A, Okazaki I, Muramatsu M, Kinoshita K, Begum NA, Nakajima T, Saito $\mathrm{H}$ and Honjo T: A target selection of somatic hypermutations is regulated similarly between $\mathrm{T}$ and $\mathrm{B}$ cells upon activation-induced cytidine deaminase expression. Proc Natl Acad Sci USA 102: 4506-4511, 2005.

14. Harris RS, Petersen-Mahrt SK and Neuberger MS: RNA editing enzyme APOBEC1 and some of its homologs can act as DNA mutators. Mol Cell 10: 1247-1253, 2002.

15. Okazaki IM, Hiai H, Kakazu N, Yamada S, Muramatsu M, Kinoshita $\mathrm{K}$ and Honjo T: Constitutive expression of AID leads to tumorigenesis. J Exp Med 197: 1173-1118, 2003.

16. Chan TS, Lakhchaura BD and Hsu TF: Differences in deoxycytidine metabolism in mouse and rat. Biochem J 210: 367-371, 1983.

17. Miwa M, Eda H, Ura M, Ouchi KF, Keith DD, Foley LH and Ishitsuka H: High susceptibility of human cancer xenografts with higher levels of cytidine deaminase to a 2'-deoxycytidine antimetabolite, 2'-deoxy-2'-methylidenecytidine. Clin Cancer Res 4: 493-497, 1998.

18. Chen EH, Johnson EE II, Vetter SM and Mitchell BS: Characterization of the deoxycytidine kinase promoter in human lymphoblast cell lines. J Clin Invest 95: 1660-1668, 1995.

19. Hapke DM, Stegmann AP and Mitchell BS: Retroviral transfer of deoxycytidine kinase into tumor cell lines enhances nucleoside toxicity. Cancer Res 56: 2343-2347, 1996. 